\title{
Building fracton phases by Majorana manipulation
}

\author{
Yizhi You $\oplus^{1}$ and Felix von Oppen ${ }^{2,3}$ \\ ${ }^{1}$ Princeton Center for Theoretical Science, Princeton University, Princeton, New Jersey 08544, USA \\ ${ }^{2}$ Dahlem Center for Complex Quantum Systems and Fachbereich Physik, Freie Universität Berlin, 14195 Berlin, Germany \\ ${ }^{3}$ Institute of Quantum Information and Matter, California Institute of Technology, Pasadena, California 91125, USA
}

(Received 22 February 2019; revised manuscript received 25 July 2019; published 16 August 2019)

\begin{abstract}
Fracton topological phases host fractionalized topological quasiparticles with restricted mobility, with promising applications to fault-tolerant quantum computation. While a variety of exactly solvable fracton models have been proposed, there is a need for platforms to realize them experimentally. We show that a rich set of fracton phases emerges in interacting Majorana band models whose building blocks are within experimental reach. Specifically, our Majorana constructions overcome a principal obstacle, namely, the implementation of the complicated spin cluster interactions underlying fracton stabilizer codes. The basic building blocks of the proposed constructions include Coulomb blockaded Majorana islands and weak interisland Majorana hybridizations. This setting produces a wide variety of fracton states and promises numerous opportunities for probing and controlling fracton phases experimentally. Our approach also reveals the relation between fracton phases and Majorana fermion codes and further generates a hierarchy of fracton spin liquids.
\end{abstract}

DOI: 10.1103/PhysRevResearch.1.013011

\section{INTRODUCTION}

Searching for and exploring exotic phases of matter is a principal goal of condensed matter physics. In the presence of strong interactions, quantum many-body systems composed of a limited number of elementary particles assume a remarkable variety of exotic phases whose low-energy degrees of freedom are much richer than suggested by their constituents. Prominent examples of such emergent quantum phases are topological phases, whose quasiparticle excitations carry fractional quantum numbers and obey anyonic statistics $[1,2]$. The low-energy properties of these topologically ordered states are characterized by topological quantum field theories (TQFTs) $[3,4]$.

Recently, distinct long-range entangled states, transcending the TQFT paradigm and termed fracton phases, have been discovered and intensively studied in exactly solvable lattice models [5-17]. Fracton topological order shares many features with topological order, including nontrivial braiding statistics and symmetry fractionalization [12,15,17-32]. At the same time, fracton phases have a subextensive groundstate degeneracy depending on system size in addition to lattice topology, and quasiparticles with restricted mobility, moving within lower-dimensional manifolds such as planes, lines, or fractals [5-11,13,23,24,26,33-37]. The subdimensional nature of fracton excitations gives rise to unconventional features including glassiness and subdiffusive dynamics $[9,38]$. The restricted quasiparticle mobility makes fracton

Published by the American Physical Society under the terms of the Creative Commons Attribution 4.0 International license. Further distribution of this work must maintain attribution to the author(s) and the published article's title, journal citation, and DOI. stabilizer codes interesting for quantum memories and quantum computation [5,10,12,17-30,35,38-43].

While theoretical aspects of fracton phases have been intensively explored via quantum stabilizer codes as well as higher-rank gauge theories, their physical realization remains a key challenge $[6,11,13]$. The principal obstacle is that models exhibiting fracton physics tend to be based on involved spin cluster interactions. This raises the question whether and how such exotic fracton states emerge in models with more physical ingredients and interactions. These might then be amenable to experimental implementation and, assuming tunable interaction parameters, allow for controlling and manipulating fracton phases and excitations, e.g., for quantum computing.

Here we show that many known fracton stabilizer codes can be obtained from Majorana-band models with strong on-site interactions. There is currently a major push to develop the required ingredients for realizing such models in the context of Majorana-based quantum computing [44], and our work shows how to apply these developments to fracton phases of matter. Our constructions include fracton phases of both flavors, referred to as type-I and type-II fracton codes. While excitations of the former are created by line or membrane operators, excitations of type-II fractons are generated by operators with fractal support.

The principal ingredients of our constructions are Majorana hybridization as well as interactions which fix local fermion parities [45-51]. These interactions can be implemented using Majorana islands, also referred to as Majorana Cooper pair boxes, which underlie current designs for Majorana-based topological qubits [44,45,52,53]. Each island contains some number of Majoranas, e.g., at the ends of semiconductor wires proximity coupled to a superconductor $[44,54,55]$. The island's charging energy fixes its fermion parity, corresponding to a multi-Majorana interaction. In order 
(a)

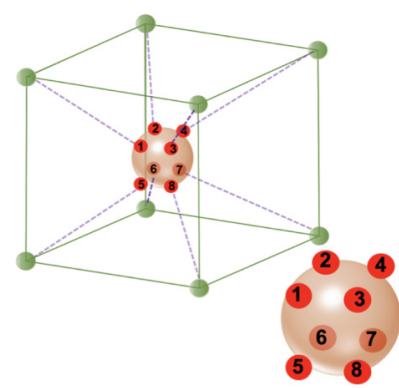

(b)

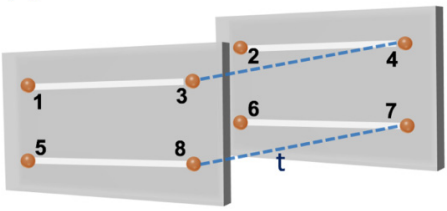

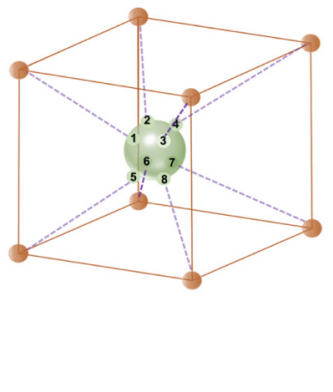

(c)

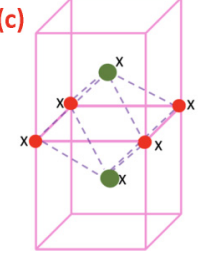

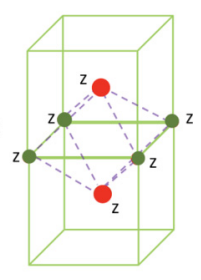

FIG. 1. Construction for the planon-lineon code. (a) Bodycentered-cubic lattice with eight Majorana zero modes on all corner (green) and center (red) sites. Majorana hybridization is illustrated by dashed lines. (b) Setup for realizing the Majorana quartet interaction in Eq. (2). Two Majorana quartets (red dots) are placed on floating superconducting islands, fixing the corresponding fermion parities via charging energy. The third quartet in Eq. (2) is generated by the Majorana hybridizations indicated by the dashed lines. (c) The two types of octahedral cells which support the stabilizers of the planon-lineon code.

to realize fracton phases, the fermion parity typically has to be fixed for overlapping sets of Majoranas. We show how such interactions can be realized by judiciously hybridizing Majoranas from multiple Majorana islands.

Our construction opens new avenues in the study of fracton phases from both conceptual and applied perspectives. On the theoretical side, our Majorana-based setups for fracton models imply that fractonic phases of matter can emerge from strongly interacting one-dimensional $p$-wave superconductors. Moreover, they expose the close relation between threedimensional (3D) Majorana fermion codes [56] and fracton codes. On the experimental side, our Majorana-based models not only provide a possible route towards realizing fracton phases of matter, but might also give access to interesting observables of fracton phases and their phase transitions. In particular, the Hamiltonian can be tuned away from the stabilizer limit to explore confinement and disorder effects on fracton matter.

\section{PLANON-LINEON CODE}

\section{A. Majorana representation}

We begin with each site of a body-centered-cubic lattice containing eight Majoranas $\gamma^{1}, \ldots, \gamma^{8}$ which are each hybridized with a Majorana on a nearest-neighbor site as shown in Fig. 1. Thus, the Hamiltonian is

$$
H=-i t^{\prime} \sum_{\langle i, j\rangle}\left(\gamma_{i}^{1} \gamma_{j}^{7}+\gamma_{i}^{2} \gamma_{j}^{8}+\gamma_{i}^{4} \gamma_{j}^{5}+\gamma_{i}^{3} \gamma_{j}^{6}\right)
$$

and can be thought of as built from crossing one-dimensional Kitaev chains along the $( \pm 1, \pm 1,1)$ directions.

We now consider on-site interactions which couple quartets of Majoranas,

$$
H_{\mathrm{int}}=U\left(\gamma_{i}^{1} \gamma_{i}^{3} \gamma_{i}^{8} \gamma_{i}^{5}+\gamma_{i}^{3} \gamma_{i}^{4} \gamma_{i}^{7} \gamma_{i}^{8}+\gamma_{i}^{4} \gamma_{i}^{2} \gamma_{i}^{6} \gamma_{i}^{7}\right),
$$

and suppress hopping of single Majoranas between sites. In the strong- $U$ limit, they project each site into the $\gamma_{i}^{1} \gamma_{i}^{3} \gamma_{i}^{8} \gamma_{i}^{5}=$ $\gamma_{i}^{3} \gamma_{i}^{4} \gamma_{i}^{7} \gamma_{i}^{8}=\gamma_{i}^{4} \gamma_{i}^{2} \gamma_{i}^{6} \gamma_{i}^{7}=-1$ subspace. The product of the three parity constraints also implies $\gamma_{i}^{2} \gamma_{i}^{1} \gamma_{i}^{5} \gamma_{i}^{6}=-1$, constraining the Majorana quartets associated with the four vertical faces of the red cube in Fig. 1.

Under these parity constraints, each site retains a single spin-1/2 degree of freedom. We can choose the parities of the top and bottom faces as the Pauli-Z operator $\sigma_{i}^{z}=\gamma_{i}^{1} \gamma_{i}^{2} \gamma_{i}^{4} \gamma_{i}^{3}$ and the product of two Majoranas on any vertical edge as the Pauli- $X$ operator $\sigma_{i}^{x}$, or vice versa. In the strong- $U$ limit, the Majorana hybridizations are a perturbation. The leading-order Hamiltonian involves 16-Majorana terms for the octahedra in Fig. 1 and becomes

$$
H=-\sum_{\text {octahedra }}\left\{\prod_{i \in \text { octa }^{a}} \sigma_{i}^{x}+\prod_{i \in \text { octa }^{b}} \sigma_{i}^{z}\right\}
$$

in the spin representation. Here octa ${ }^{a}$ and octa ${ }^{b}$ refer to the two types of octahedra in Fig. 1 with red (green) sites at top and bottom and four green (red) sites in between. Thus, our construction exactly reproduces the planon-lineon model in Ref. [10] whose elementary quasiparticles are lineons and planons with mobility restricted to the $z$ direction and the $x z$ (yz) planes, respectively.

\section{B. Experimental implementation}

To implement this construction with interacting Majoranas, we must establish that one can realize the on-site interaction in Eq. (2). To this end, we distribute the eight Majoranas of each site over two adjacent superconducting islands (SCIs) (see Fig. 1). Each SCI could be made from two semiconductor quantum wires proximity coupled to the same superconductor. The proximity-coupled quantum wires effectively realize open Kitaev chains with two Majorana zero modes localized at their ends, so there is a total of four Majoranas on each SCI. By virtue of their charging energy, each SCI can be tuned to have even fermion parity, effectively implementing the interaction terms $U\left(\gamma_{i}^{1} \gamma_{i}^{3} \gamma_{i}^{8} \gamma_{i}^{5}+\gamma_{i}^{4} \gamma_{i}^{2} \gamma_{i}^{6} \gamma_{i}^{7}\right)$ in Eq. (2) $[52,53]$.

To generate the remaining four-Majorana interaction in Eq. (2), we turn on interisland Majorana hybridization $H_{t}=$ it $\left(\gamma^{3} \gamma^{4}+\gamma^{8} \gamma^{7}\right)$ with amplitude $t$. Note that at the same time, there is no hybridization between Majoranas $\gamma_{i}^{1}$ and $\gamma_{i}^{2}$ as well as $\gamma_{i}^{5}$ and $\gamma_{i}^{6}$. These interisland hybridizations can in principle be implemented by direct tunnel coupling. Alternatively, one can bridge between the two Majorana islands using a coherent link [52]. Such a link consists of an additional proximitycoupled quantum wire whose fermion parity is fixed by its charging energy. The two Majorana end states would then be tunnel coupled to the two Majoranas of the Majorana islands which one wants to hybridize. Since the hybridization between the Majoranas on the coherent link and the islands can be realized through gate-controlled tunnel junctions, the hybridization strength is tunable. The same hybridization 
via coherent link can be used to implement the Majorana hybridizations between different sites as given by Eq. (1).

When a large charging energy fixes the fermion parities of the SCIs, single-Majorana tunneling between the islands is suppressed and the lowest-order processes involve pairs of Majorana tunneling terms. The leading-order Hamiltonian is

$$
H_{\text {eff }}=U\left(\gamma_{i}^{1} \gamma_{i}^{3} \gamma_{i}^{8} \gamma_{i}^{5}+\gamma_{i}^{4} \gamma_{i}^{2} \gamma_{i}^{6} \gamma_{i}^{7}\right)+\frac{c t^{2}}{U} \gamma_{i}^{3} \gamma_{i}^{4} \gamma_{i}^{7} \gamma_{i}^{8},
$$

with $c$ a number of order one. This produces an anisotropic version of the on-site interaction in Eq. (2). The anisotropy does not modify the ground-state manifold and thus the lowenergy spin- $1 / 2$ degree of freedom. The additional weak hybridization $t^{\prime}$ of Majoranas on nearest-neighbor sites as described by Eq. (1) generates the spin interactions on the octahedra. As a sufficient condition, the local spin- $1 / 2$ degrees of freedom remain intact in the limit $U>t>t^{\prime}$ such that $t^{2} / U>t^{\prime}$, and the resulting effective Hamiltonian realizes the planon-lineon code at leading order.

\section{FRACTAL CHAMON CODE}

Following this construction, one can realize many fracton codes based on crossing Kitaev chains and strong on-site interactions. We show now how a type-II fracton code, the fractal Chamon code, emerges in this manner. Unlike type-I fracton codes whose excitations are created by straight-line or planar membrane operators, excitations of type-II fracton codes involve operators with fractal support $[5,34]$. For the fractal Chamon code [57], excitations exist at corners of 2D Sierpinski triangles of side length $2^{n}$. (Note that we define type-II fracton codes as having excitations which are created by operators with fractal support. We do not require the stricter condition that all excitations are generated by such fractal operators.) These corner excitations are immobile in the $x y$ plane unless one enlarges the Sierpinski triangle to side length $2^{n+1}$ which is inhibited by a large energy barrier.

The configuration of crossing Kitaev chains in Fig. 2 generates a hexagonal-close-packed lattice, with each site containing eight Majoranas. Six of these labeled $\gamma^{1}, \ldots, \gamma^{6}$ are paired to Majoranas on nearest-neighbor sites in the same $x y$ plane, while the remaining two (labeled $\gamma^{A}$ and $\gamma^{B}$ ) pair with Majoranas on neighboring sites along the $\pm z$ direction.

Now consider the on-site interaction

$$
H=U\left(\gamma^{1} \gamma^{2} \gamma^{4} \gamma^{3}+\gamma^{1} \gamma^{2} \gamma^{6} \gamma^{5}+\gamma^{5} \gamma^{6} \gamma^{B} \gamma^{A}\right) .
$$

For strong coupling, the eight Majoranas retain a spin-1/2 degree of freedom. As for the planon-lineon model, we can define spin operators through $\sigma_{x}=\gamma_{1} \gamma_{3} \gamma_{5} \gamma_{B}=\gamma_{2} \gamma_{4} \gamma_{6} \gamma_{A}$ and $\sigma_{z}=i \gamma_{1} \gamma_{2}=i \gamma_{3} \gamma_{4}=i \gamma_{5} \gamma_{6}$. With these definitions, the lowenergy Hamiltonian takes the form of the fractal Chamon code with five-qubit stabilizers defined on prisms as illustrated in Fig. 2 [57]. The elementary excitations include lineons with mobility restricted along the $z$ direction, generated by a line of $\sigma_{z}$ operators, in addition to the fractal excitations at the corners of 2D Sierpinski triangles within the $x y$ planes.

The on-site interaction in Eq. (5) can be implemented as for the planon-lineon code. The Majoranas involved in $\gamma^{1} \gamma^{2} \gamma^{3} \gamma^{4}$ and $\gamma^{A} \gamma^{B} \gamma^{5} \gamma^{6}$ are placed onto two floating SCIs as in Fig. 2. The charging energy of each SCI fixes their
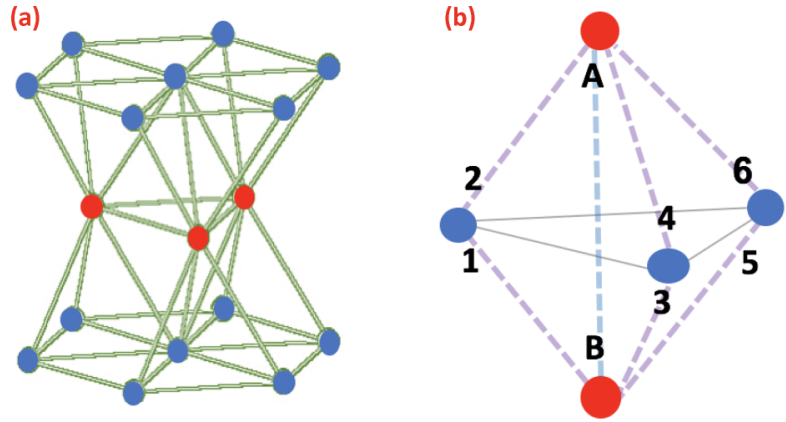

(c)
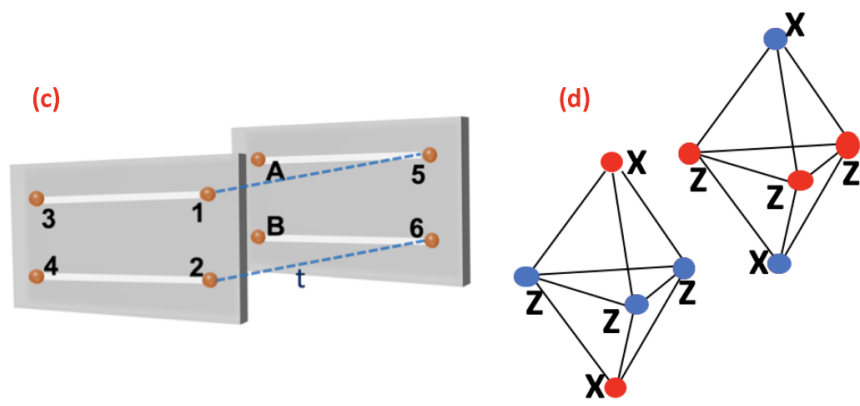

FIG. 2. Majorana construction for the fractal Chamon code. (a) Hexagonal close-packed lattice with eight Majoranas per site with elementary prism. (b) The dashed purple and blue lines illustrate Majorana hybridizations for $\gamma^{1}, \ldots, \gamma^{6}$ and $\gamma^{A}, \gamma^{B}$, respectively. (c) On-site projection is implemented by placing Majorana wires on floating superconducting islands with interisland tunneling and strong charging energy. (d) The effective Hamiltonian after projection becomes a sum of five-spin stabilizers on the elementary prisms.

fermion parity. Hybridizations it $\left(\gamma_{i}^{1} \gamma_{i}^{5}+\gamma_{i}^{2} \gamma_{i}^{6}\right)$ effectively generate the remaining four-Majorana interaction $\gamma_{i}^{1} \gamma_{i}^{5} \gamma_{i}^{2} \gamma_{i}^{6}$, thereby exactly reproducing the interaction in Eq. (5).

\section{OCTAHEDRAL CHAMON CODE}

We now consider a system of hybridized Majoranas on a face-centered-cubic lattice with 12 Majoranas on each site. Each of these Majoranas, placed on the edges of a cube, pairs with their partner on one of the nearest-neighbor sites as illustrated in Fig. 3.

For each site, we place the 12 Majoranas onto three SCIs as shown in Fig. 3. The charging energy of the SCIs fixes the fermion parities $\eta^{1} \eta^{2} \eta^{9} \eta^{12}=\eta^{4} \eta^{3} \eta^{10} \eta^{11}=\eta^{7} \eta^{6} \eta^{5} \eta^{8}=-1$. Weak tunneling between the different SCIs,

$$
H_{t^{\prime}}=i t\left(\eta^{1} \eta^{4}+\eta^{12} \eta^{11}+\eta^{9} \eta^{5}+\eta^{7} \eta^{10}\right),
$$

generates the effective Majorana interactions $\eta^{1} \eta^{4} \eta^{12} \eta^{11}$ and $i \eta^{9} \eta^{5} \eta^{7} \eta^{10} \eta^{12} \eta^{11}$. These interactions project each site into a single spin- $1 / 2$ degree of freedom and the corresponding Hamiltonian becomes

$$
H=-\sum_{\text {octahedra }} \sigma_{r+e_{x}}^{x} \sigma_{r-e_{x}}^{x} \sigma_{r+e_{z}}^{z} \sigma_{r-e_{z}}^{z} \sigma_{r+e_{y}}^{y} \sigma_{r-e_{y}}^{y},
$$

which is just the octahedral Chamon code [9].

\section{CUBOCTAHEDRON FRACTON CODE}

We now consider the same structure of crossing Kitaev chains as in Eq. (6), yielding a face-centered-cubic lattice. At 


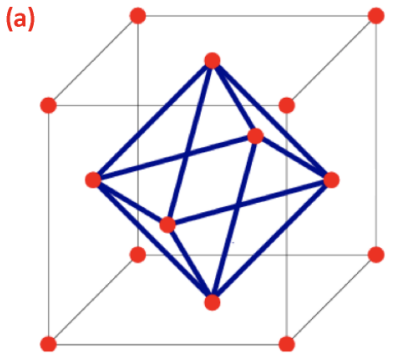

(b)
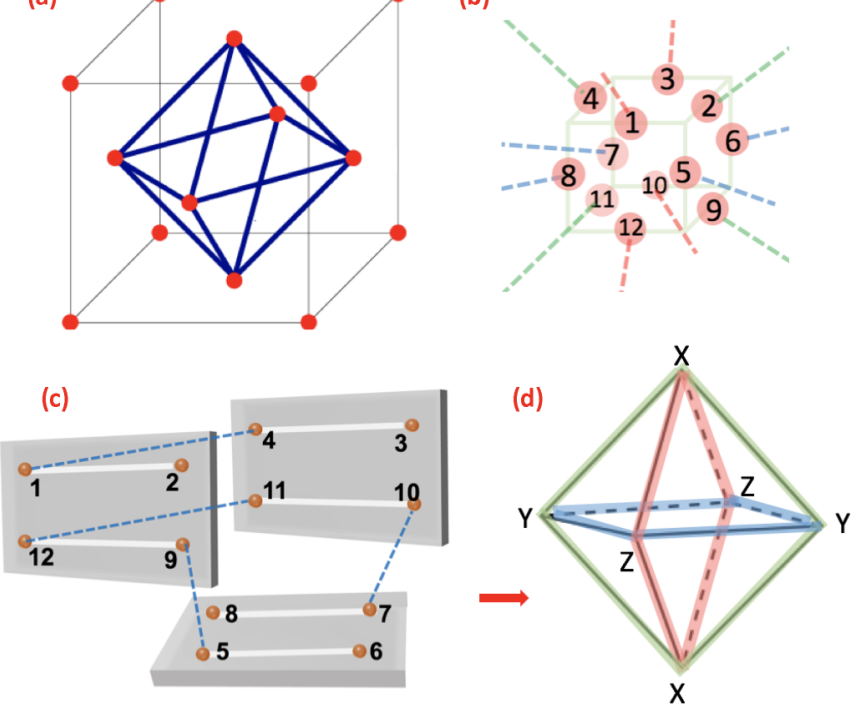

FIG. 3. Majorana model for the octahedral Chamon code. (a) Face-centered-cubic lattice with 12 Majoranas per site. (b) Labeling and hybridization of Majoranas on one of the sites. (c) Strong on-site interactions, realizable by this arrangement of floating superconducting islands, project each site into a spin- $1 / 2$ degree of freedom. (d) Stabilizers of the low-energy Hamiltonian exist on the octahedra and the effective Hamiltonian becomes the octahedral Chamon code.

each site, there are 12 Majoranas, each of which pairs with a partner at a nearest-neighbor site.

We place the 12 on-site Majoranas onto three SCIs as shown in Fig. 4. Their charging energies fix the fermion parities $\eta^{5} \eta^{6} \eta^{7} \eta^{8}=\eta^{1} \eta^{3} \eta^{10} \eta^{12}=\eta^{2} \eta^{4} \eta^{11} \eta^{9}=-1$. Turning on tunneling between the SCIs,

$$
H_{t^{\prime}}=i t\left(\eta^{5} \eta^{1}+\eta^{12} \eta^{8}+\eta^{3} \eta^{4}+\eta^{9} \eta^{6}\right)
$$

generates effective Majorana interactions $\eta^{1} \eta^{5} \eta^{8} \eta^{12}$ and $i \eta^{3} \eta^{4} \eta^{8} \eta^{12} \eta^{9} \eta^{6}$. When combined with the parity fixing for each SCI, these interactions project each site into a single spin-1/2 degree of freedom and the Hamiltonian becomes the cuboctahedron code as shown in Fig. 4. Each stabilizer is a 12 -spin interaction on the cuboctahedra. (The cuboctahedra are made of six corner-sharing plaquettes from the $x y, y z$, and $x z$ planes.) The 12-spin term on the cuboctahedron originates from the product of the 24 Majorana pairs on the hinges. This fracton code belongs to the family of type-I fracton codes whose quasiparticles have restricted mobility. Similar to the Chamon code, the ground-state degeneracy on a three-torus depends on the greatest common divisor of the system size.

The construction of the cuboctahedron code shares many similarities with the octahedral Chamon code in Eq. (7). The geometry of this Majorana network corresponds to four intersecting plaquettes sharing a corner at a site. The four-Majorana projections $\gamma^{1} \gamma^{3} \gamma^{10} \gamma^{12}, \gamma^{5} \gamma^{6} \gamma^{7} \gamma^{8}$, and $\gamma^{2} \gamma^{9} \gamma^{11} \gamma^{4}$ produce three intersecting Wen-plaquette models on the $x y, y z$, and $x z$ planes labeled by spin qubits $\left(X^{1}, Z^{1}\right)$, $\left(X^{2}, Z^{2}\right)$, and $\left(X^{3}, Z^{3}\right)$. The remaining four- and six-Majorana interactions couple the three intersecting Wen-plaquette models via an anyon condensate. By imposing $Y_{1} Y_{2} Y_{3}=1$ and

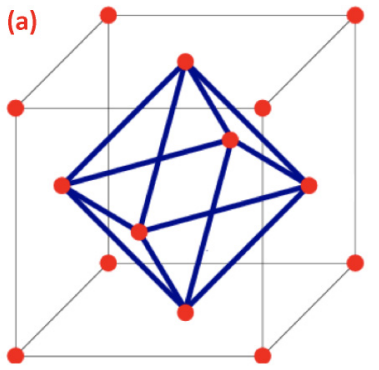

(b)
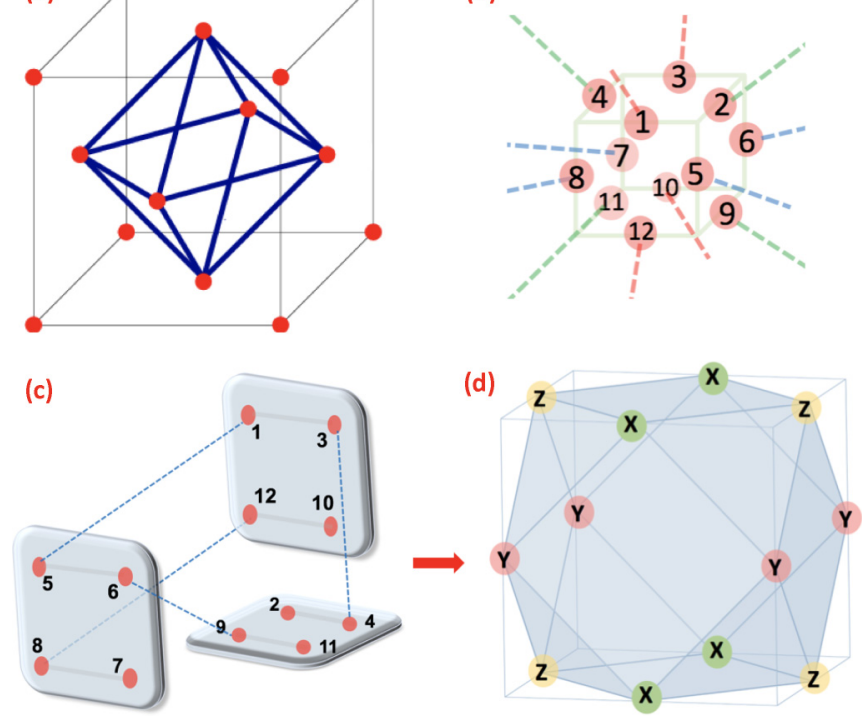

FIG. 4. Majorana construction for the cuboctahedron code. (a) Underlying face-centered-cubic lattice. (b) Interactions project each site into a spin-1/2 degree of freedom. (c) SCI design for implementing the on-site interactions. (d) The effective spin Hamiltonian is a cuboctahedron fracton code. Each stabilizer is a 12-spin interaction on the cuboctahedron. The cuboctahedron is made of six corner-sharing plaquettes from the $x y, y z$, and $x z$ planes.

$Z_{1} X_{2}=1$ on each site, the three spin qubits are reduced to one Pauli spin degree of freedom represented by $\sigma^{x}=$ $X_{1} Z_{2}, \sigma^{y}=X_{2} Z_{3}$, and $\sigma^{z}=X_{3} Z_{1}$. The three corner-sharing intersecting Wen-plaquette models form an octahedron which exactly reproduces the Chamon code in Eq. (7). Likewise, if we impose $Y_{1} Y_{2} Y_{3}=1$ and $X_{1} Z_{2}=1$ on each site, the three spin qubits are reduced to one Pauli spin degree of freedom represented by $\bar{\sigma}^{x}=Z_{1} X_{2}, \bar{\sigma}^{y}=Z_{2} X_{3}$, and $\bar{\sigma}^{z}=Z_{3} X_{1}$. This exactly reproduces the cuboctahedron structure which ties the six Wen-plaquette models on the six plaquette faces of the cuboctahedron. En route, our construction also reveals the relation between coupled (2D) toric code layers and the $3 \mathrm{D}$ Chamon code via an anyon condensate.

\section{TRIANGLE ISING MODEL WITH FRACTAL SYMMETRY BREAKING}

Symmetries are indispensable for characterizing different phases of matter. Typically, one deals with global symmetries, whose operations act extensively on the entire volume of the system. Fractal subsystem symmetries, which act only on a subset of sites whose number scales with linear size $L$ as $L^{d}$ with some fractal dimension $d$ have attracted much attention with the recent developments on fracton topological order. Most notably, systems with such symmetries appear in the context of glassiness. Examples are the triangle and tetrahedral Ising models, whose Hamiltonians have fractal $Z_{2}$ symmetry when flipping spins on arbitrary Sierpinski triangles. We now show how to utilize a crossing Majorana network to generate the 2D triangle Ising model whose lowenergy ground states exhibit fractal symmetry breaking [34]. 

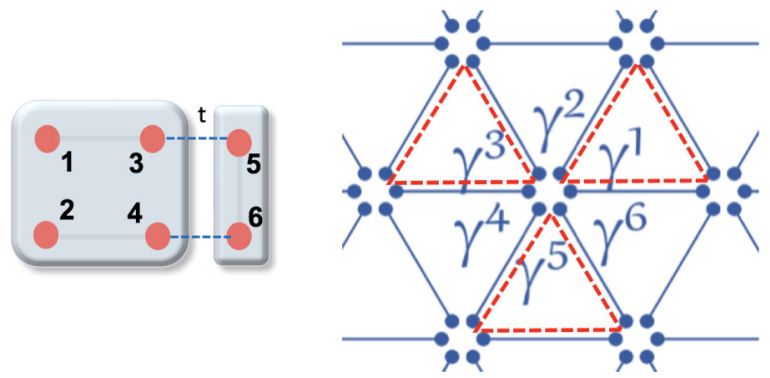

FIG. 5. Shown on the right are crossing Kitaev wires yielding a triangle lattice with six Majoranas per site. On the left we place four Majoranas on one SCI and the other two on the other. The SCI holding four Majoranas is subject to a charging energy with fixed fermion parity. Majorana tunneling between the two SCIs is indicated by dashed lines. This effectively produces the on-site interaction in Eq. (9).

Consider a Majorana network on the honeycomb lattice with six Majoranas per site and each Majorana hybridized with a Majorana on a nearest-neighbor site as shown in Fig. 5. This structure resembles three crossing Kitaev wires at angles $\theta=2 N \pi / 3$. We impose the on-site interaction

$$
H=-U\left(\eta_{i}^{1} \eta_{i}^{2} \eta_{i}^{3} \eta_{i}^{4}+\eta_{i}^{3} \eta_{i}^{4} \eta_{i}^{5} \eta_{i}^{6}+\eta_{i}^{1} \eta_{i}^{2} \eta_{i}^{5} \eta_{i}^{6}\right)
$$

In the large- $U$ limit, the interaction enforces $\eta_{i}^{1} \eta_{i}^{2} \eta_{i}^{3} \eta_{i}^{4}=$ $\eta_{i}^{3} \eta_{i}^{4} \eta_{i}^{5} \eta_{i}^{6}=\eta_{i}^{1} \eta_{i}^{2} \eta_{i}^{5} \eta_{i}^{6}=1$. As the third term is the product of the first two, these are two independent constraints and the six site Majoranas are projected into a spin-1/2 subspace. The resultant Hamiltonian involves the products of the three Majorana pairs in the upward triangle. Written in the spin basis, the Hamiltonian reduces to the triangle Ising model,

$$
H=\sum_{\nabla} \prod_{i \in \nabla} \sigma_{i}^{z}
$$

This Hamiltonian is invariant under fractal $Z_{2}$ transformations which flip the spins on arbitrary Sierpinski triangles. At zero temperature, the ground state breaks this fractal symmetry as characterized by the three-point correlator

$$
\left\langle\sigma_{i}^{z}\left(r_{0}\right) \sigma_{i}^{z}\left(r_{0}+a e_{r}\right) \sigma_{i}^{z}\left(r_{0}+a e_{r^{\prime}}\right)\right\rangle .
$$

This correlation function does not vanish at long wavelengths. However, it fluctuates and becomes nonzero only when the three points hit the corners of a large Sierpinski triangle with distance $a=2^{n} l_{0}$. This fractal structure breaks the discrete scale invariance.

To implement the on-site fermion interaction in Eq. (9), we place four and two Majoranas on one SCI each as shown in Fig. 5. While the two-Majorana island is grounded, the SCI with four Majoranas is floating so that its charging energy fixes the parity $\eta^{1} \eta^{2} \eta^{3} \eta^{4}=-1$. Weak tunneling it $\left(\eta^{3} \eta^{5}+\right.$ $\left.\eta^{4} \eta^{6}\right)$ between the islands effectively creates the other fourMajorana interaction $\eta^{3} \eta^{4} \eta^{5} \eta^{6}$.

\section{FRACTONS FROM MAJORANA FERMION CODES}

Our constructions suggest that many bosonic fracton models are equivalent to 3D Majorana fermion codes [56]. Enlarging each site of the bosonic model into a cell, the stabilizers
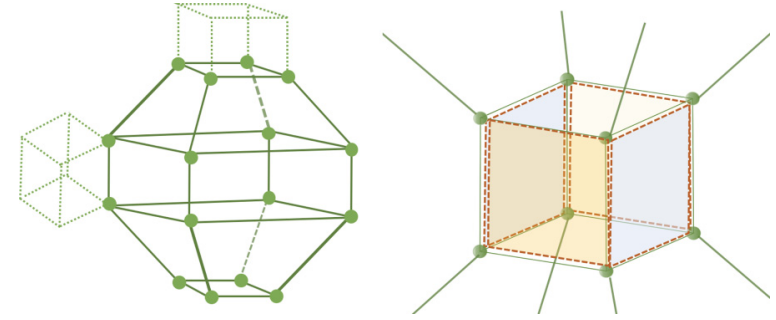

FIG. 6. On the left the truncated octahedron shares a face with the top/bottom cubes and shares a hinge with the side cube. On the right the stabilizer is defined on the four side faces of the cube (yellow and blue), as well as the entire truncated-octahedron cell.

can be expressed as products over Majoranas located on corners of certain cells or plaquettes, provided that similar to color codes [58], the plaquettes and cells share an even number of Majoranas.

We make this argument explicit for the planon-lineon code in Fig. 1. We replace each site by a small cube with eight Majoranas at its corners. The octahedra are then replaced by truncated octahedra (Fig. 6) which share a face with cubes below or above and a hinge with a side cube. The spin Hamiltonian in Eq. (2) is replaced by the Majorana fermion code

$$
H=-\sum\left\{\prod_{i \in \text { octa }} \gamma_{i}+\prod_{i \in P^{x z}} \gamma_{i}+\prod_{i \in P^{y z}} \gamma_{i}\right\} .
$$

The first term is a product over the 16 Majoranas at the corners of a truncated octahedron. The remaining terms involve products of four Majoranas on the side plaquettes $(x z$ or $y z$ ) of the cube. This Majorana fermion code defines a commuting-projector Hamiltonian which exactly reproduces the ground-state manifold of the planon-lineon code.

The same stratagem works for the other fracton codes in this paper. While a wide variety of 3D Majorana fermion codes exhibiting $Z_{2} \times Z_{2} \times Z_{2}$ order have been proposed [56], our construction suggests that numerous fracton topological orders can also be represented by Majorana fermion codes akin to color codes. In particular, this reveals the equivalence between Majorana fracton codes and bosonic fracton codes.

Now imagine we have four Majoranas per site, with each flavor forming a Majorana fermion code as given in Eq. (12). By imposing a strong on-site interflavor interaction $U \gamma_{1} \gamma_{2} \gamma_{3} \gamma_{4}$, each site is reduced to a spin-1/2 degree of freedom. The resulting spin Hamiltonian is the $\mathrm{SO}(3)$ invariant fracton spin liquid

$$
H=-\sum_{a=x, y, z}\left\{\prod_{i \in \mathrm{octa}} \sigma_{i}^{a}+\prod_{i \in P^{x z}} \sigma_{i}^{a}+\prod_{i \in P^{y z}} \sigma_{i}^{a}\right\} .
$$

One can also take $2 N$ Majoranas per site and apply interflavor interactions to obtain an $\mathrm{SO}(2 N-1)$ invariant Hamiltonian. To be concrete, we place $2 N$ Majoranas on each site, with each flavor forming a Majorana fermion code as given in Eq. (8). By imposing a strong on-site interflavor interaction $U \gamma_{1}, \ldots, \gamma_{2 N}$, each site is constrained to even fermion parity and the corresponding low-energy Hilbert space is reduced to spin- $(2 N-1) / 2$ degrees of freedom. One can use the Clifford 


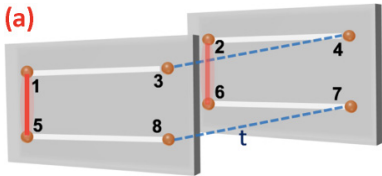

$\sigma_{x}=i \gamma_{1} \gamma_{5}$

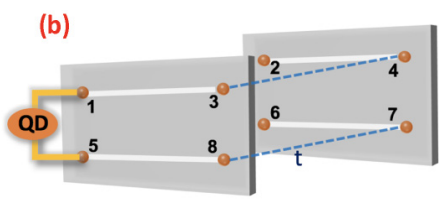

FIG. 7. Measuring and manipulating fracton phases. (a) Adding an on-site Majorana hybridization applies an effective Zeeman field to the low-energy spin degree of freedom associated with a site. (b) Coupling the Majoranas to a single level quantum dot shifts the quantum dot level energy by an amount which depends on the Majorana parity associated with the bilinear. Thus, spectroscopy of the energy shift provides a measurement of the Majorana bilinear. Similar schemes can be implemented when the spin component is represented by a product of four Majoranas.

algebra for the $\operatorname{Sp}(2 N-1)$ representation

$$
\Gamma_{a}=i \gamma_{1} \gamma_{a+1} \quad(a=1, \ldots, 2 N-1),
$$

and the resulting spin Hamiltonian is the $\operatorname{SO}(2 N-1)$ invariant spin liquid

$$
H=-\sum_{a=1, \ldots, 2 N-1}\left\{\prod_{i \in \text { octa }} \Gamma_{i}^{a}+\prod_{i \in P^{x z}} \Gamma_{i}^{a}+\prod_{i \in P_{y z}} \Gamma_{i}^{a}\right\}
$$

yielding fractonic behavior.

\section{DISCUSSION}

We have demonstrated the emergence of fracton phases of matter in interacting Majorana band models. On the theoretical side, our construction exposes the relation between fracton models and Majorana fermion codes. By extension, such Majorana fermion codes enable us to generate a hierarchy of fracton spin liquids with $\mathrm{SO}(N)$ symmetry. In addition to the examples discussed here explicitly, interacting Majoranas can also realize additional fracton models in particular and topological phases in general, including the checkerboard model proposed in Ref. [59], the 3D toric code, and the $\mathrm{X}$-cube model (see the Appendix).

On the experimental side, this provides a platform for exploring these novel strongly interacting phases. Initial experiments may focus on small fracton codes for which the ground-state degeneracy as a function of linear dimension or boundary stabilizers would be a characteristic observable. For larger system sizes, it is also interesting to go beyond realizing the exactly solvable stabilizer Hamiltonians of fracton codes. Sufficiently strong transverse fields applied to the local spin degrees of freedom drive the fracton phase into a confined phase [60]. In our platform, such transverse fields are readily implemented by adding local Majorana hybridization terms as illustrated in Fig. 7, providing access to these phases and the intervening phase transition. While fracton phases lack a local order parameter, their phases and phase transitions are characterized by nonlocal string or membrane order parameters. For instance, the expectation values of certain planar Wilson loop operators may obey perimeter or area laws depending on the phase [60]. Such expectation values can be read out by repeated preparation of a ground state and subsequent projective measurements of, say, the $\sigma_{z}$ components of all involved sites. Averaging the results for the string operators over the repeated measurements then provides access to the desired expectation value. Such projective measurements of local spins correspond to the measurement of local two- or four-Majorana parities for which several readout schemes have been proposed [52,53]. A promising scheme to measure, say, a two-Majorana parity couples a single-level quantum dot to the two Majoranas. The resulting energy shift of the quantum dot level will then depend on the Majorana parity. This is illustrated in Fig. 7. The same scheme can be extended to measure four-Majorana parities [52].

In addition to static expectation values, one can also extract dynamic correlation functions of the fracton codes from linear-response measurements. Applying a time-dependent transverse field and reading out the spins at a later time provides access to spin-spin correlation functions of the fracton code. The dynamic properties of 3D fracton codes are particularly interesting due to their glassy dynamics which results from the restricted quasiparticle mobility. Moreover, disorder in the stabilizer flip energies or the transverse fields would would allow for studies of many-body localization. Both kinds of disorder are easily tunable in this setting as intersite hybridization and on-site stabilizer energies are controllable via gate-tuned coherent links.

\section{ACKNOWLEDGMENTS}

This work was supported in part by the PCTS Fellowship (Y.Y.) and by CRC 183 of the Deutsche Forschungsgemeinschaft (F.v.O.) and was initiated at the Aspen Center for Physics, which is supported by National Science Foundation Grant No. PHY-1607611. One of us (F.v.O.) is grateful for sabbatical support from the IQIM, an NSF physics frontier center funded in part by the Moore Foundation.

\section{APPENDIX: THREE-DIMENSIONAL TORIC CODE AND X-CUBE MODEL}

Following the strategy in the main text, one can readily obtain the 3D toric code and the X-cube model from a similar Majorana construction. As pointed out in Ref. [14], both models can be obtained via coupled layer constructions based on the 2D toric code. Moreover, the 2D toric code can be obtained via a Majorana network construction [45,59].

We briefly review the Majorana construction for the 2D toric code. Consider a Majorana model on a square lattice with four Majoranas per site. Each Majorana hybridizes with its closest neighbor, as shown in Fig. 8 and described by the Hamiltonian

$$
H=-i t \sum_{j}\left(\gamma_{j}^{1} \gamma_{j+e_{r}}^{3}+\gamma_{j}^{2} \gamma_{j+e_{r}^{\prime}}^{4}\right) .
$$

Here the lattice sites are enumerated by $j$ and connected by lattice vectors $e_{r}$ and $e_{r}^{\prime}$. Each site can be viewed as a SCI whose charging energy fixes the site's fermion parity $\gamma_{j}^{1} \gamma_{j}^{2} \gamma_{j}^{3} \gamma_{j}^{4}=-1$. The low-energy Hilbert space reduces to an effective spin- $1 / 2$ degree of freedom on each site for which one can define Pauli operators (see, e.g., [59]). Treating the Majorana hybridizations as perturbations, the leading-order Hamiltonian involves Majorana hopping terms around all 
(a)

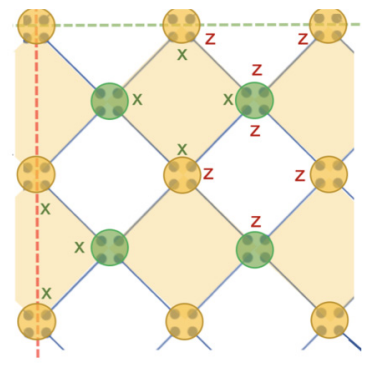

(b)

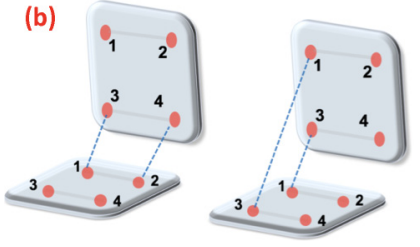

FIG. 8. Majorana constructions for the X-cube model and 3D toric code. (a) Toric code as a low-energy theory for an interacting Majorana band model. Each site supports a spin 1/2 and the plaquette operators involve products of $\sigma^{z}\left(\sigma^{x}\right)$ for white (yellow) plaquettes. (b) Majorana interactions effectively implementing the coupled-layer construction. Each link of the cubic lattice has two SCIs with four Majoranas per island which form 2D toric codes on the $i j$ layers. On the left the interaction $H_{A}$ leads to the 3D X-cube model. On the right the interaction $H_{B}$ leads to the 3D toric code. (c) Cubic lattice with two SCIs on each link.

elementary plaquettes. In terms of spin operators, the plaquette terms form a checkerboard pattern and become $\prod_{\square} \sigma_{i}^{z} \sigma_{j}^{z} \sigma_{k}^{z} \sigma_{l}^{z}$ and $\prod_{\square} \sigma_{i}^{x} \sigma_{j}^{x} \sigma_{k}^{x} \sigma_{l}^{x}$ for white and yellow plaquettes, respectively (see Fig. 8). This is just the toric (or surface) code.

We can now implement the coupled layer construction by placing two SCIs on each link of a cubic lattice. Each SCI hosts four Majoranas forming a 2D toric code on each $i j$ layer. The model resembles decoupled 2D toric codes with each $i$ link holding qubits from the $i j$ and $i k$ layers as shown in Fig. 8.

We can now add additional tunneling terms between Majoranas on the same site but different SCIs (see Fig. 8). We consider two separate cases, described by the two Hamiltonians

$$
\begin{aligned}
& H_{A}=-i t\left(\gamma_{a}^{1} \gamma_{b}^{3}+\gamma_{a}^{2} \gamma_{b}^{4}\right), \\
& H_{B}=-i t\left(\gamma_{a}^{1} \gamma_{b}^{3}+\gamma_{a}^{3} \gamma_{b}^{1}\right) .
\end{aligned}
$$

Here $a$ and $b$ label the two SCIs on the same site. These tunneling terms, together with the fixed fermion parities of each SCI, generate effective on-site $X X$ or $Z Z$ interactions between spins from perpendicular layers, but on the same link. This couples the perpendicular 2D toric code layers. In the strong-coupling limit, the interaction $H_{B}$ leads just to the $3 \mathrm{D}$ toric code model

$$
H_{\mathrm{eff}}=\sum\left\{\prod_{i \in \text { vertex }} \sigma_{i}^{x}+\prod_{i \in \text { plaquette }} \sigma_{i}^{z}\right\}
$$

The Hamiltonian involves six-spin vertex interactions involving $\sigma^{z}$ and four-spin plaquette interactions via $\sigma^{x}$. This model exhibits $3 \mathrm{D} Z_{2}$ topological order with nontrivial particle loop braiding. Likewise, the interaction $H_{A}$ leads to the 3D X-cube model

$$
H_{\mathrm{eff}}=\sum\left\{\prod_{i \in v^{i j}} \sigma_{i}^{x}+\prod_{i \in \text { cube }} \sigma_{i}^{z}\right\} .
$$

[1] X.-G. Wen, Int. J. Mod. Phys. B 4, 239 (1990).

[2] R. Willett, J. P. Eisenstein, H. L. Störmer, D. C. Tsui, A. C. Gossard, and J. H. English, Phys. Rev. Lett. 59, 1776 (1987).

[3] X.-G. Wen and A. Zee, Phys. Rev. B 46, 2290 (1992).

[4] R. Dijkgraaf and E. Witten, Commun. Math. Phys. 129, 393 (1990).

[5] J. Haah, Phys. Rev. A 83, 042330 (2011).

[6] G. B. Halász, T. H. Hsieh, and L. Balents, Phys. Rev. Lett. 119, 257202 (2017).

[7] S. Vijay, J. Haah, and L. Fu, Phys. Rev. B 94, 235157 (2016).

[8] S. Vijay, J. Haah, and L. Fu, Phys. Rev. B 92, 235136 (2015).

[9] C. Chamon, Phys. Rev. Lett. 94, 040402 (2005).

[10] W. Shirley, K. Slagle, and X. Chen, arXiv:1806.08625.

[11] K. Slagle and Y. B. Kim, Phys. Rev. B 96, 165106 (2017).

[12] H. Ma, E. Lake, X. Chen, and M. Hermele, Phys. Rev. B 95, 245126 (2017).

[13] T. H. Hsieh and G. B. Halász, Phys. Rev. B 96, 165105 (2017).

[14] S. Vijay, arXiv:1701.00762.

[15] K. Slagle and Y. B. Kim, Phys. Rev. B 96, 195139 (2017).
[16] D. J. Williamson, Phys. Rev. B 94, 155128 (2016).

[17] H. Ma, A. T. Schmitz, S. A. Parameswaran, M. Hermele, and R. M. Nandkishore, Phys. Rev. B 97, 125101 (2018).

[18] M. Pretko and L. Radzihovsky, Phys. Rev. Lett. 120, 195301 (2018).

[19] H. Ma, M. Hermele, and X. Chen, Phys. Rev. B 98, 035111 (2018).

[20] D. Bulmash and M. Barkeshli, Phys. Rev. B 97, 235112 (2018).

[21] K. Slagle and Y. B. Kim, Phys. Rev. B 97, 165106 (2018).

[22] W. Shirley, K. Slagle, Z. Wang, and X. Chen, Phys. Rev. X 8, 031051 (2018).

[23] A. Prem, S. Vijay, Y.-Z. Chou, M. Pretko, and R. M. Nandkishore, Phys. Rev. B 98, 165140 (2018).

[24] K. Slagle, A. Prem, and M. Pretko, arXiv:1807.00827.

[25] A. Gromov, Phys. Rev. Lett. 122, 076403 (2019).

[26] S. Pai and M. Pretko, Phys. Rev. B 97, 235102 (2018).

[27] M. Pretko, Phys. Rev. B 95, 115139 (2017).

[28] H. Ma and M. Pretko, Phys. Rev. B 98, 125105 (2018).

[29] M. Pretko, Phys. Rev. B 96, 035119 (2017).

[30] M. Pretko, Phys. Rev. B 96, 115102 (2017). 
[31] Y. You, T. Devakul, F. J. Burnell, and S. L. Sondhi, Phys. Rev. B 98, 035112 (2018).

[32] Y. You, T. Devakul, F. Burnell, and S. L. Sondhi, arXiv:1805.09800.

[33] T. Devakul, Y. You, F. J. Burnell, and S. L. Sondhi, SciPost Phys. 6, 007 (2019).

[34] B. Yoshida, Phys. Rev. B 88, 125122 (2013).

[35] M. Pretko, Phys. Rev. B 98, 115134 (2018).

[36] H. Song, A. Prem, S.-J. Huang, and M. A. Martin-Delgado, Phys. Rev. B 99, 155118 (2019).

[37] A. Gromov, arXiv:1812.05104.

[38] A. Prem, J. Haah, and R. Nandkishore, Phys. Rev. B 95, 155133 (2017).

[39] A. Y. Kitaev, Ann. Phys. (NY) 303, 2 (2003).

[40] D. Bulmash and M. Barkeshli, arXiv:1806.01855.

[41] A. Prem, M. Pretko, and R. M. Nandkishore, Phys. Rev. B 97, 085116 (2018).

[42] D. Bulmash and T. Iadecola, Phys. Rev. B 99, 125132 (2019).

[43] W. Shirley, K. Slagle, and X. Chen, SciPost Phys. 6, 041 (2019).

[44] R. M. Lutchyn, E. P. A. M. Bakkers, L. P. Kouwenhoven, P. Krogstrup, C. M. Marcus, and Y. Oreg, Nat. Rev. Mater. 3, 52 (2018).

[45] S. Vijay, T. H. Hsieh, and L. Fu, Phys. Rev. X 5, 041038 (2015).

[46] M. Barkeshli and J. D. Sau, arXiv:1509.07135.

[47] Z. Nussinov, G. Ortiz, and E. Cobanera, Phys. Rev. B 86, 085415 (2012).
[48] L. A. Landau, S. Plugge, E. Sela, A. Altland, S. M. Albrecht, and R. Egger, Phys. Rev. Lett. 116, 050501 (2016).

[49] E. Sagi, H. Ebisu, Y. Tanaka, A. Stern, and Y. Oreg, Phys. Rev. B 99, 075107 (2019).

[50] C. Wille, R. Egger, J. Eisert, and A. Altland, Phys. Rev. B 99, 115117 (2019).

[51] A. Thomson and F. Pientka, arXiv:1807.09291.

[52] T. Karzig, C. Knapp, R. M. Lutchyn, P. Bonderson, M. B. Hastings, C. Nayak, J. Alicea, K. Flensberg, S. Plugge, Y. Oreg, C. M. Marcus, and M. H. Freedman, Phys. Rev. B 95, 235305 (2017).

[53] S. Plugge, A. Rasmussen, R. Egger, and K. Flensberg, New J. Phys. 19, 012001 (2017).

[54] R. M. Lutchyn, J. D. Sau, and S. Das Sarma, Phys. Rev. Lett. 105, 077001 (2010).

[55] Y. Oreg, G. Refael, and F. von Oppen, Phys. Rev. Lett. 105, 177002 (2010)

[56] S. Bravyi, B. Leemhuis, and B. M. Terhal, Ann. Phys. (NY) 326, 839 (2011).

[57] C. Castelnovo and C. Chamon, Philos. Mag. 92, 304 (2012).

[58] H. Bombin and M. A. Martin-Delgado, Phys. Rev. Lett. 97, 180501 (2006).

[59] Y. You, D. Litinski, and F. von Oppen, Phys. Rev. B 100, 054513 (2019).

[60] T. Devakul, S. A. Parameswaran, and S. L. Sondhi, Phys. Rev. B 97, 041110(R) (2018). 